${ }^{1}$ Servicio de Medicina Interna. Hospital Universitario Clínico San Carlos. Madrid, España. ${ }^{2}$ Servicio de Farmacología clínica. Hospital Universitario Clínico San Carlos. Madrid, España. ${ }^{3}$ Servicio de Urgencias. Hospital Universitario Clínico San Carlos. Madrid, España. Trabajo no recibió financiamiento.

Los autores declaran no tener conflictos de interés.

Recibido el 11 de enero de 2018, aceptado el 30 de abril de 2019 .

Correspondencia a: Jorge Carriel Mancilla jorge.carriel@salud.madrid.org

\section{Síndrome de Klinefelter diagnosticado en un adulto a raíz del estudio de infección respiratoria recurrente y masa mediastínica. Caso clínico}

\author{
JORGE CARRIEL MANCILLA ${ }^{1}$, ANTONIO LEONE $^{2}$, \\ ENCARNACIÓN BORREGUERO MARTÍNEZ ${ }^{1}$, PEDRO RUIZ ARTACHO $^{3}$, \\ FERNANDO TORNERO ROMERO ${ }^{1}$, ELPIDIO CALVO MANUEL ${ }^{1}$
}

\section{Thymoma in a patient with klinefelter syndrome. Case report}

\begin{abstract}
Klinefelter syndrome (47, XXY in most cases) is a frequently underdiagnosed chromosomal anomaly associated with multiple comorbidities in adult life. Patients with Klinefelter syndrome have a higher risk of cancer. Specifically, these patients have a higher risk for mediastinal germ cell tumors. It is estimated that $8 \%$ of male patients with mediastinal tumors have Klinefelter. We report a 42-years-old male who suffered recurrent respiratory infections. During the study, a mediastinal mass was found, whose pathological study disclosed a type $B$ thymoma. The patient had a history of infertility, high stature, gynecomastia, obesity with gynecoid distribution of body fat and testicular atrophy. A karyotype was requested (47, XXY), confirming the diagnosis of Klinefelter syndrome.
\end{abstract}

(Rev Med Chile 2019; 147: 518-521)

Key words: Genetics, Medical; Klinefelter Syndrome; Mediastinal Diseases; Neoplasms, Germ Cell and Embryonal.
$\mathrm{E}$ 1 síndrome de Klinefelter es una cromosomopatía (47,XXY en la mayoría de los casos) que se asocia a múltiples comorbilidades en la vida adulta tales como bronquitis crónica, bronquiectasias, enfisema pulmonar, tumores de células germinales, cáncer de mama, venas varicosas en extremidades inferiores, osteoporosis, prolapso mitral, diabetes mellitus, entre otras ${ }^{1}$. Existe controversias respecto a si los pacientes con Klinefelter tienen riesgo elevado de tumores sólidos, encontrándose la incidencia de tumores de células germinales en mediastino claramente aumentada ${ }^{2,3}$. La asociación genética Klinefelter/ masa mediastínica aún se desconoce y es sujeto de investigación. Presentamos el caso de un varón de 42 años diagnosticado de síndrome de Klinefelter, a raíz del hallazgo de infecciones respiratorias de repetición y masa mediastínica anterior.

\section{Caso clínico}

Varón de 42 años que consulta en nuestro centro por cuadro de $72 \mathrm{~h}$ de evolución caracterizado por fiebre, tos y expectoración. Es la tercera ocasión en los últimos 2 años que consulta por infección respiratoria, objetivándose en la placa de tórax una consolidación de lóbulo inferior derecho. En la radiografía actual se aprecia adyacente al infiltrado una imagen sugerente de masa mediastínica anterior. En placas previas de los últimos 6 años se objetiva dicha imagen, que ha aumentado de tamaño de forma progresiva hasta alcanzar un diámetro aproximado de $7 \times 7 \mathrm{~cm}$ (Figura 1).

Respecto a los antecedentes personales, refiere haber tenido tuberculosis en la niñez, sin otra patología infecciosa o comorbilidades relevantes. 


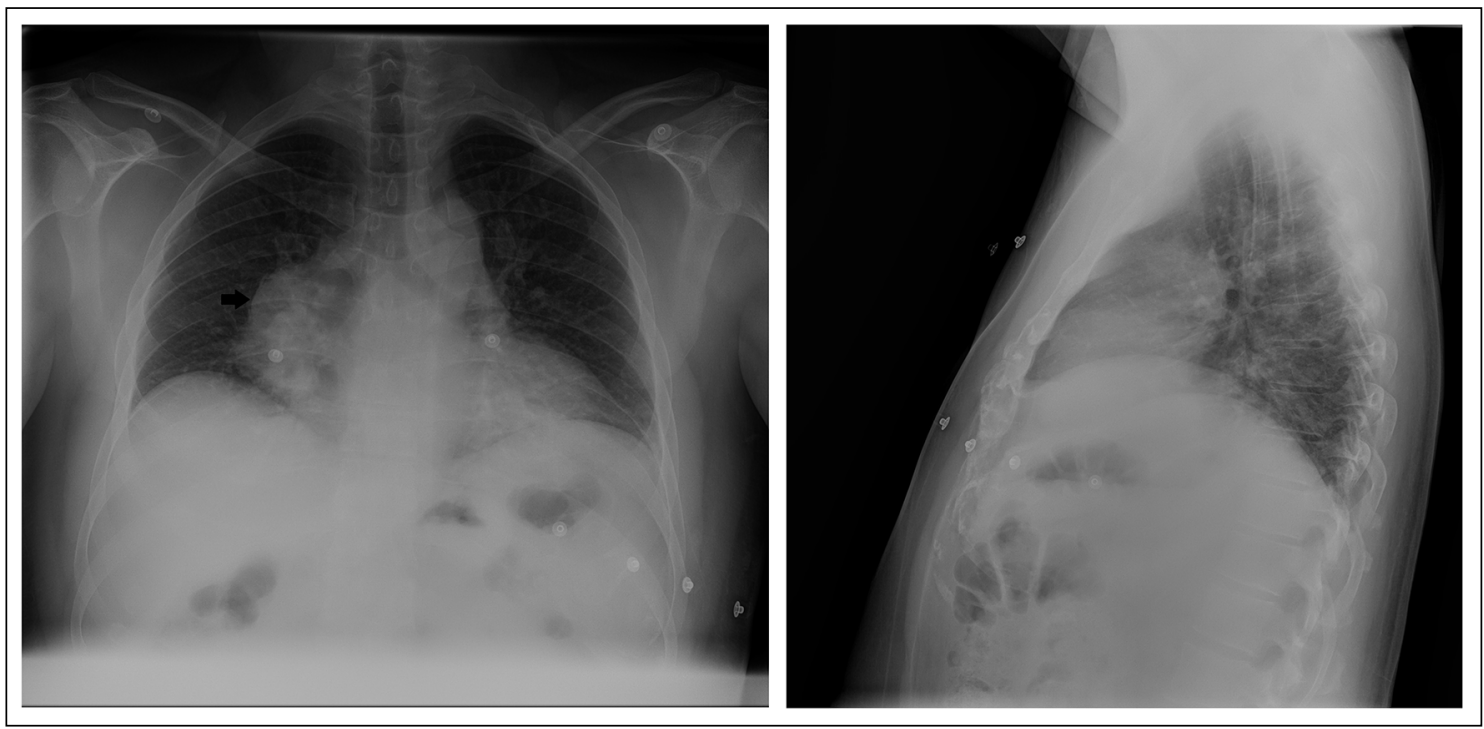

Figura 1.

Sin embargo, se encuentra en seguimiento junto con su pareja por la Unidad de Fertilidad debido a problemas para concebir. Al revisar su historia clínica, en los análisis previos solicitados por dicha unidad, se objetiva aumento de hormonas gonadotropas [FSH $33 \mathrm{UI} / \mathrm{L}$ (rango normal 1,0-12,0 $\mathrm{UI} / \mathrm{L}$ ), LH $22 \mathrm{UI} / \mathrm{L}$ (rango normal 2,0-12,0 UI/L)], con disminución de testosterona de $7 \mathrm{nmol} / \mathrm{L}$ (rango normal 9,36-37,10 nmol/L), por lo que es diagnosticado de hipogonadismo primario. El paciente se encuentra pendiente de realizar un espermiograma al momento de nuestra valoración.

A la exploración física destacan talla alta $(1,9 \mathrm{~m})$, obesidad (IMC $\left.32 \mathrm{~kg} / \mathrm{m}^{2}\right)$ con distribución ginecoide de la grasa corporal, ginecomastia, escaso vello corporal, atrofia testicular y pene pequeño de $2 \mathrm{~cm}$.

Se le solicita TAC de tórax en el que se describe masa mediastínica anterior derecha, con diámetro anteroposterior máximo de $6,9 \mathrm{~cm}$, de contorno polilobulado, sólida, con calcificaciones toscas en su interior y con áreas de menor atenuación centrales (Figura 2). Estos hallazgos podrían estar en relación con un teratoma, otro tipo de tumor germinal, o timoma. Se solicitan anticuerpos anti-receptor de acetilcolina, alfafetoprotenía, gonadotrofina coriónica humana, $\beta 2$-microglobulina, CA 19.9, CA 125 y CA 15.3, encontrándose todos los parámetros en rango de la normalidad.

Debido al fenotipo descrito, infertilidad e hi- pogonadismo hipergonadotrófico en un varón con masa mediastínica en estudio se solicita estudio citogenético por sospecha de síndrome de Klinefelter. En el cariograma se observa un cromosoma $\mathrm{X}$ adicional, lo que confirma este diagnóstico, siendo su cariotipo: 47,XXY.

El caso fue presentado en sesión multidisciplinar de patología torácica, optando por toma de biopsia con aguja gruesa de la lesión. El resultado histopatológico fue timoma tipo B. Posteriormente se realizó extracción quirúrgica de la masa, siendo el diagnóstico definitivo de la lesión timoma tipo B2.

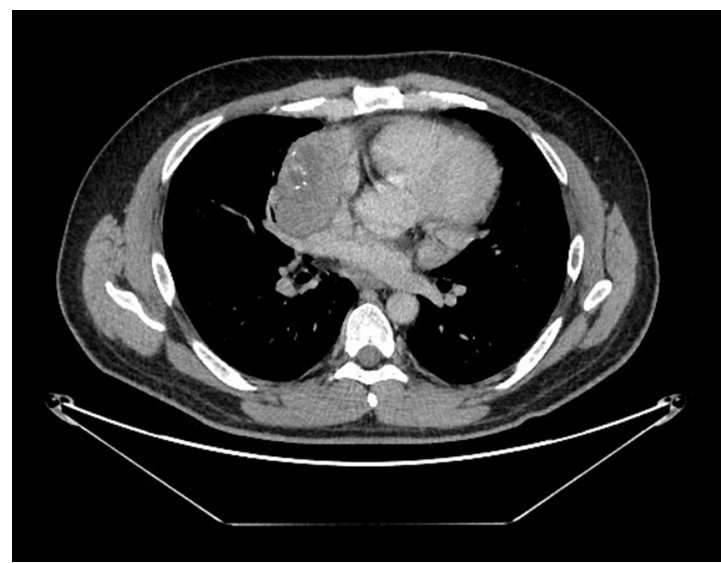

Figura 2. 


\section{Discusión}

El síndrome de Klinefelter es el trastorno de los cromosomas sexuales más frecuente en los varones con una prevalencia estimada de 1:500 a 1:1.000 nacidos vivos ${ }^{2-4}$. Sin embargo, es subdiagnosticado, siendo la edad media de diagnóstico de 33 años $^{2,5}$. En países como Dinamarca se confirman citogenéticamente menos de 30\% del número esperado de casos anuales ${ }^{6}$. En dicho país, la frecuencia de Klinefelter con diagnóstico citogenético disminuye al aumentar la distancia al laboratorio citogenético más cercano y aumenta en áreas cercanas a laboratorios con un interés especial en su diagnóstico ${ }^{6}$.

Esta condición fue descrita por primera vez en 1942 por Harry F. Klinefelter, a raíz de la valoración de 9 hombres adultos que compartían características clínicas tales como ginecomastia, pene pequeño y azooespermia, junto con la presencia de un cromosoma $\mathrm{X}$ adicional ${ }^{5}$. El cariotipo clásico $(47, \mathrm{XXY})$ es usualmente resultado de una no-disyunción meiótica durante la gametogénesis parental, tanto de origen paterno (53\%) como materno (44\%), siendo únicamente $3 \%$ de casos producto de un error en la división postcigótica resultando en mosaicismo ${ }^{7}$.

Tras una búsqueda sistemática en MEDLINE, LILACS y SciELO con las palabras síndrome de Klinefelter, tumor mediastínico, timoma, teratoma y cromosomopatía, incluyendo publicaciones entre los años 2008 y 2018, con texto en idioma español e inglés, encontramos menos de 50 artículos publicados sobre el tema, pese a utilizar múltiples combinaciones de dichas palabras. Se realizó además una búsqueda de casos en nuestro centro desde 2005, que incluyó diagnóstico de síndrome de Klinefelter, timoma, teratoma o masa mediastínica, encontrando únicamente un caso previo de Klinefelter asociado a teratoma retroperitoneal.

Los resultados de la cohorte sueca publicada en 2016 por Ji y cols. muestran que los pacientes con Klinefelter tienen riesgo de cáncer similar a la población general, sin embargo proporcionalmente, presentan riesgo disminuido para tumores sólidos (excepto tumores de mama), y riesgo aumentado para leucemia y linfoma no Hodgkin ${ }^{2}$. La evidencia epidemiológica disponible actualmente resulta inequívoca para confirmar que los pacientes con Klinefelter son especialmente susceptibles a desa- rrollar 3 tipos de tumores: linfoma no Hodgkin, cáncer de mama y tumores de estirpe germinal extragonadal $l^{6-8}$.

Respecto a los tumores de células germinales, en la cohorte danesa publicada por Hasle, se objetivó que los pacientes con Klinefelter tienen un riesgo relativo de 66,7 (IC 95\% de 18-171) de presentar tumores en mediastino, habitualmente de estirpe germinal ${ }^{6}$. Se estima que $8 \%$ de los pacientes varones con tumores mediastínicos tienen síndrome de Klinefelter, y también se han reportado tumores de células germinales asociados a Klinefelter en retroperitoneo y cavidad abdominal ${ }^{8}$. La hipótesis más ampliamente aceptada para explicar la presencia de estos tumores es una migración incompleta o errónea de las células germinales del saco vitelino hacia la cresta geni$\mathrm{tal}^{7,8}$. Debido a estos antecedentes, la presencia de tumor mediastínico de estirpe germinal en varones jóvenes debe acarrear alta sospecha de síndrome de Klinefelter, y por tanto se debe realizar siempre estudio citogenético ${ }^{8}$.

A raíz de la presentación de este caso, consideramos que hay que prestar especial atención a la exploración física de los varones jóvenes con masa mediastínica buscando signos clínicos que sugieran Klinefelter, y recomendamos solicitar siempre cariotipo a aquellos pacientes diagnosticados de tumor de estirpe germinal. El fenotipo del síndrome de Klinefelter suele pasar desapercibido si no se tiene un alto nivel de sospecha.

\section{Referencias}

1. Bojesen A, Juul S, Birkebaek NH, Gravholt CH. Morbidity in Klinefelter syndrome: a Danish register study based on hospital discharge diagnoses. J Clin Endocrinol Metab 2006; 91: 1254-60.

2. Ji J, Zöller B, Sundquist J, Sundquist K. Risk of solid tumors and hematological malignancy in persons with Turner and Klinefelter syndromes: A national cohort study. Int J Cancer 2016; 139: 754-8.

3. De Sanctis V, Fiscina B, Soliman A, Giovannini M, Yassin M. Klinefelter syndrome and cancer: from childhood to adulthood. Ped Endocrinol Rev 2013; 11 (1): 44-50.

4. Nussbaum R, McInnes R, Willard H. Sex chromosome aneuploidy. En: Nussbaum R, editor. Thompson \& Thompson Genetics in Medicine. 8va edición. Philadelphia. Elsevier; 2016. p. 95-7. 
5. Song JS, Lee SH, Jin DK, Kim SH. A case report of rare $\mathrm{XXY} / \mathrm{XX}$ mosaicism in a phenotypic male with Klinefelter syndrome and mediastinal germ cell tumor. Genet Couns 2014; 25 (2): 215-20.

6. Hasle H, Mellemgaard A, Nielsen J, Hansen J. Cancer incidence in men with Klinefelter syndrome. Br J Cancer 1995; 71: 416-20.
7. Völkl T, Langer T, Aigner T, Greess J, Rauch A, Dörr H. Klinefelter syndrome and Mediastinal Germ Cell Tumors. Am J Med Genet A 2006; 140A: 471-81.

8. Aguirre D, Nieto K, Lazos M, Peña R, Palma I, Kofman-Alfaro S, Queipo G. Extragonadal germ cell tumors are often associated with Klinefelter syndrome. Hum Pathol 2006; 37: 477-80. 